\title{
La ciudad de Zamora en la Edad Media. Reseña y balance historiográfico*1
}

\author{
Manuel Fernando Ladero Quesada \\ UNED
}

\begin{abstract}
RESUMEN
ABSTRACT

En este trabajo se analiza la producción In this report, the historiographic historiográfica sobre la ciudad de Zamora en la Edad Media a lo largo de la segunda mitad del pasado siglo. Se señalan las principales lineas de investigación cultivadas, las lagunas aun existentes y se valora criticamente un buen número de los trabajos mencionados. Se incluye un amplio anexo bibliográfico. production of the city of Zamora in the Middle Ages in the second part of Twenty Century is analised. There are named the main lines of the investigation, the blanks that still existing and the critical values of a certain number about the mencionated reports. There is also included a wide bibliographic appendix.

PALABRAS CLAVE

Zamora. Edad Media. Historiografía.

KEY WORDS

Zamora, Middle Ages, Historiography.

\footnotetext{
- Este trabajo fue presentado como ponencia en el II Congreso de Historia de Zamora, celebrado en dicha ciudad a finales del año 2003, con el titulo de Los estudios sobre la Zamora medieval. Varios son los motivos que me mueven ha incluirlo ahora en este volumen de homenaje. El primero. naturalmente, el hecho de que aún permanece inédito porque las Actas de dicho Congreso todavia no se han publicado. En segundo lugar, porque su temática encaja plenamente en una de las lineas de investigación que cultivo el profesor Martín a lo largo de su dilatada trayectoria profesional y que, me consta. le era especialmente querida. Por último, y sin duda la razón más importante, el que su elaboración fue fruto de un encargo personal del profesor Martín. Allá donde esté confio en que no se sienta demasiado decepcionado por los resultados y que, la última vez de tantas, sea indulgente con su díscolo amigo y companero.

' Los números entre corchetes remiten al anexo bibliográfico. La ponencia limita su atención a la investigación sobre la ciudad de Zamora y su tierra e instituciones muy ligadas a ella (cabildo, monasterios, algunas jurisdicciones señoriales, etc) y obras generales de referencia. No se aborda pues el comentario de la historiografia relativa a otros concejos de realengo o señorios enclavados en la actual provincia como Toro, Villalpando, Villafáfila o Benavente y condado de Benavente que cuentan. sobre todo estos últimos, con un bagaje investigador suficiente como para merecer una reseña específica. Para Toro véanse los trabajos de Alba Lopez. para Villalpando los de Vaca Lorenzo y Calvo Lozano, para Villafáfila los de Rodríguez Rodríguez, para Benavente los de Fuentes Ganzo. Hernández Vicente, Maceda Cortés y
} la clásica historia de Ledo del Pozo y para el Condado los de Becerro Pita.
\end{abstract}


«Ojalá, queridísimo amigo, pudiera saludarte en persona» (Juan Gil de Zamora, Dictaminis epithalamium. Cap. VI)

Hace quince años, uno de los participantes en el Primer Congreso de Historia de Zamora, se refería en su aportación al mismo al «abismo historiográfico» con el que el historiador medievalista se encontraba a la hora de encontrar apoyaturas escritas anteriormente para apuntalar o fundamentar sus conclusiones o hipótesis.

Era una observación muy atinada que, sin duda, todos los que nos hemos ocupado en las últimas décadas de los temas históricos zamoranos, compartimos absolutamente y corroboramos. Porque, una vez hecha la obligada y justa cita de los intelectuales decimonónicos como Ursicinio Álvarez [9] o Cesáreo Fernández Duro $^{2}[29,30]$, ponderando su ingente labor y la todavia hoy indudable autoridad y utilidad de sus escritos, la realidad es que el panorama historiográfico de las seis primeras décadas del siglo $x x$, se nos presenta como un enorme desierto en el que apenas unos cuantos oasis salvan a la Zamora medieval de caer en el olvido.

Efectivamente, hasta la década de los setenta el vacío resulta espectacular, sólo hay tres obras que podamos mencionar como de verdadera utilidad para el conocimiento del período medieval zamorano. Además, dos de ellas se refieren al conocimiento de las fuentes documentales, no son por tanto y en sentido estricto trabajos de investigación. Me refiero al catálogo del primitivo fondo de documentos históricos del Archivo Municipal de Zamora, publicado en 1948 por María del Carmen Pescador [106], y a la Guía-Inventario de los Archivos Zamoranos, publicada por Antonio Matilla en 1964 [98]. Eso si, ambas obras son todavia hoy de una enorme utilidad para el investigador.

El único trabajo que, en puridad, podemos calificar como estudio histórico, aunque sus concepciones metodológicas se hallen hoy notablemente superadas, y que se refiere en parte al período medieval, es el que publicó Enrique Fernández Prieto en 1953 referido a la nobleza de Zamora [31], que sienta las bases de una línea de investigación que, como más adelante comentaré, tiene todavía ante sí un amplio margen de desarrollo.

Por añadir algún título más a esta escueta nómina, cabe mencionar otro trabajo de este mismo autor referido al convento de las Dueñas, publicado en 1954 [32] y reeditado en 1988; la obra de su progenitor, José Fernández Domínguez [28], relativa al protagonismo de los territorios zamoranos en la guerra civil tras la muerte

\footnotetext{
: También cabe recordar las obras de Calvo MADRONO, I. Descripción. geográfica. historica y estadistica de la provincia de Zamora. Madrid, 1914; GARnACHO, T. M. : Breve noticia de algunas antigüedades de la ciudad y provincia de Zamora. Zamora, 1878. Gómez CarABIAS, F.: Guía sinoptica-estadística de las parroquias de las diócesis de Zamora y la vicaria de Alba y Aliste de Santiago de Compostela. Zamora, 1884. OlmEdo, F.: La provincia de Zamora. Guia geográfica e histórica. Zamora, 1905. PICATOSTE, $\checkmark$ : Descripcion e historia politica. eclesiástica y monumental de España. Provincia de Zamora. Madrid, 1869. Pinuela XimÉnez, A.: Descripción histórica de la ciudad de Zamora, su provincia y obispado. Zamora, 1987 (reimp). Zatarain Fernández, M.: Apuntes. Noticias curiosas para formalizar la historia eclesiástica de Zamora y su diócesis. Zamora, 1898.
} 
de Enrique IV, publicada en 1929 y objeto de una reedición hace diez años a cargo del Instituto de Estudios Zamoranos, muy apegada al texto de las crónicas como era habitual en la investigación histórica de esa época. También, aunque partiendo del enfoque de la historia del arte y del patrimonio, se pueden encontrar noticias de interés en los volúmenes dedicados a Zamora por Manuel Gómez Moreno [49], reeditados en 1980. Todo ello se completa con la edición que del fuero de Zamora hicieron en 1916 Américo Castro y Federico de Onís ${ }^{3}$, si bien conviene no olvidar una edición anterior realizada por Fernández Duro en sus Memorias Históricas.

A partir de 1970, la publicistica sobre la Zamora medieval comienza a experimentar un renacimiento significativo, al menos en términos comparativos con el periodo anterior. El punto de arranque lo constituye, sin duda alguna, la aparición en 1972 en la revista Hispania del estudio de Amando Represa sobre la génesis y evolución urbana de la Zamora medieval [112]. Investigación que, al igual que ocurre con algunas películas, se ha convertido en una referencia de culto. Un trabajo que formaba parte del proyecto de tesis doctoral del autor, antiguo director del Archivo de Simancas, que debía versar sobre el desarrollo urbano de las ciudades castellanas de la cuenca del Duero y que no llegó a concretarse pero que, sin embargo, supuso una aportación fundamental en el desarrollo posterior de la investigación, no sólo para el caso de Zamora sino también para otros núcleos como Palencia o Segovia. En la misma línea, hace pocos años Manuel de la Granja ha construido un sólido trabajo repleto de referencias documentales sobre la progresiva repoblación interna de la ciudad y el surgimiento de sus barrios, collaciones y pueblas [48].

Por las mismas fechas el profesor Linehan nos ofrecía la primera aproximación rigurosa a la documentación de origen eclesiástico en las páginas que dedicó al hoy archifamoso, entonces no tanto, episodio de las Dueñas de Zamora en su libro sobre la Iglesia española y el Papado en el siglo XIII [82]. Aproximación que ha culminado, casi treinta años después con la publicación de un erudito ensayo que va mucho más allá del acontecimiento concreto para presentarnos un pormenorizado fresco sobre la sociedad, la moral y las costumbres de la época [83]. En fin, si el fin justifica los medios, habrá que dar por buenos determinados actos de «filibusterismo intelectual' que el autor denuncia y que le han llevado a obsequiarnos con el fruto final de su investigación. Convengamos todos pues en la justeza de la cita final de Lord Byron con la que Linehan cierra su libro: "las cosas grandes surgen de las pequeñas». Simultáneamente a las sesiones de este Congreso ha visto la luz la última aportación del profesor Linehan -en colaboración con José Carlos de Lera- a la historiografía zamorana: una aproximación a la figura del obispo zamorano del siglo xIII don Suero Pérez, basada $\in$ n su testamento y otros documentos [84].

CASTRO, Américo y Onis, Federico de: Fueros leoneses de Zamora, Salamanca, Ledesma y Alba de Tormes. Madrid, 1916. Existe también otra edición más reciente del texto foral a cargo de MAJADA NEILA, J.: Fuero de Zamora. Salamanca, 1983. 
En el anterior congreso, el profesor Martín explicaba el origen de su dedicación a los temas zamoranos a partir de la documentación "heredada" de su maestro don Emilio Sáez. Fruto de este encargo han sido la redacción de un importante número de trabajos de variada indole sobre la sociedad y la economía de las tierras zamoranas en la plena Edad Media, que el autor ha venido publicando entre los años setenta y ochenta, recopilados en su mayor parte en un volumen editado por el centro de la UNED de Zamora [90], y la edición del primer, lamentablemente hasta la fecha único, volumen de documentos del Archivo Catedralicio que abarca hasta 1261 [89]. En su ponencia también denunciaba el hecho de que permanecieran inéditas o publicadas casi clandestinamente las investigaciones que, sobre la base del mismo fondo documental, compusieron algunos de sus, por entonces, alumnos de la Universidad de Salamanca ${ }^{4}$. Hoy, quince años después, no cabe sino reiterar esa denuncia aún con más energía, puesto que si esta situación se prolonga es claro que esas investigaciones terminarán cayendo en el olvido. Es imperativo pues hacer un nuevo llamamiento, a quien corresponda, para que esos trabajos, convenientemente actualizados si es necesario, vean por fin la luz, incluso utilizando para ello las nuevas tecnologias. En ese sentido, la creación de un soporte electrónico en red que aloje estas y otras investigaciones, catálogos y regestas documentales y cualesquier otros materiales (cartografía, fotografía, etc.) de utilidad para el estudio del pasado zamorano es no sólo una iniciativa técnicamente factible, sino económicamente viable.

En cualquier caso, la labor desarrollada por Martín y sus discipulos, además del incuestionable valor intrínseco de sus aportaciones, sirvió para poner de manifiesto a todos los investigadores la enorme riqueza documental que atesora el Archivo Catedralicio, muy superior, como él mismo señala, al de otras diócesis del entorno. Afortunadamente en la actualidad, desde su aparición en 1999, y merced a la minuciosa y profesional tarea de su autor, José Carlos de Lera, contamos con ese excelente instrumento que es el catálogo de la documentación medieval del archivo [76], que nos ofrece la verdadera dimensión de su importancia y que abre una amplia expectativa para la prosecución de las investigaciones basadas en este fondo en un futuro inmediato.

La década de los setenta contempla la aparición de algunos otros estudios de interés. En 1974 apareció en la revista Hispania un artículo de José Sánchez Herrero referido a cofradías y hospitales bajomedievales en algunas diócesis del valle del Duero, entre ellas Zamora, que es la primera aportación sistemática y sobre sólidas bases documentales que se ofrecia sobre este tipo de instituciones [120]. Años más tarde, el autor ampliaría datos sobre la cuestión en su ponencia

- Garcia Perez, J. R. y Nicolas Crispin. I.: El dominio territorial de la diócesis de Zamora, 1970. Sanchez Rodriguez, M.: Las cláusulas penales del Tumbo Negro de Zamora. 1972 Coria Couino, J. I.: La sociedad zamorana (siglos xim-xv) a traves de sus testamentos. 1977. Ferrero Ferrero, F.: Rentas del Cabildo Catedral de Zamora en el siglo xv. Analisis del libro de rentas 1446-1451. 1980. 
sobre la iglesia y la religiosidad en Zamora a finales del siglo $\mathrm{xV}$, presentada en las Jornadas sobre Zamora, su entorno y América celebradas en 1991. También sobre esta misma temática, centrada en la Edad Moderna pero con datos de interés para fines del Medievo, es obligado mencionar la tesis doctoral de la norteamericana Maureen Flynn, obra muy poco difundida en España pero de sumo interés, cuya traducción sería muy deseable [40].

En 1975 se publica la tesis doctoral de María Luisa Bueno sobre el monasterio cisterciense de Moreruela [11], institución que será objeto de un segundo estudio, con criterios metodológicos y planteamientos teóricos sensiblemente diferentes, en el que se amplia el marco cronológico y se edita la colección documental, por parte de Isabel Alfonso [8], que también constituyó su tesis doctoral y que vio la luz en 1986. Aun cabe mencionar otro trabajo sobre Moreruela cuyo autor es Manuel de la Granja y que se editó en 1990 [47].

En el año 1976 aparece la única muestra dignamente publicada de las investigaciones de Marciano Sánchez basadas en la documentación catedralicia, me refiero a su trabajo sobre las cláusulas penales del Tumbo Negro de Zamora, publicado en el homenaje a Fray Justo Pérez de Urbel [123].

Por último, para completar la glosa de lo publicado en esta década, no podemos olvidar las numerosas noticias de interés para el periodo medieval que pueden encontrarse en la investigación que sobre los gremios zamoranos llevó a cabo Maria del Carmen Pescador [108] y que apareció, en sucesivos números de la Revista de Archivos, Bibliotecas y Museos, entre los años 1968 y 1975. Y el estudio que, desde el punto de vista jurídico, analizó la estructura de la población de la Extremadura leonesa de los siglos centrales de la Edad Media, partiendo de varios textos forales, entre ellos el de Zamora, obra de Maria Teresa Gacto [42] aparecida en 1977.

En los años ochenta, además del progresivo aumento de los trabajos publicados, asistimos a un hecho de trascendencia notable para el mejor conocimiento del pasado medieval de la ciudad y su tierra; me refiero a la ampliación de las líneas de investigación a cuestiones relativas a lo que podriamos denominar «sociedad civil», partiendo de documentación de origen no eclesiástico.

Quedaron mencionadas ya las sucesivas aportaciones del profesor Martín basadas en documentos catedralicios, a ellas habría que añadir los artículos publicados por Marciano Sánchez en Studia Zamorensia, especialmente el referido a una interesante reglamentación sobre aceñas del siglo XIV $[125,127,128]$. El estudio de Guadalupe Beraza sobre la biblioteca del cabildo en el siglo xIII [51] y algunas aportaciones nuestras relativas al proceso de diferenciación de los respectivos patrimonios del obispo y el cabildo y a la presentación y descripción de otro de los libros tumbos conservados en el mencionado archivo, el denominado Tumbo Tercero con documentación de los siglos XIV y XV relativa a las propiedades urbanas del cabildo $[57,55]$. José Carlos de Lera analizó de nuevo el contenido de dicho libro en una comunicación presentada en el I Congreso de Historia de Za- 
mora. Con todo, y en relación con el estudio del mundo eclesiástico, todavía está pendiente la investigación monográfica de algunas instituciones monásticas de enorme importancia en la vida de la ciudad y su tierra como Montamarta o Valparaíso y otras agrupaciones conventuales.

De la labor desarrollada por los profesores Moreta y Vaca y un grupo de sus alumnos del extinto Colegio Universitario, surgieron las primeras publicaciones que tomaron como base la documentación del Archivo Histórico Provincial. Los primeros, en un trabajo conjunto aparecido en el número de 1982 de la revista Agricultura y Sociedad [101], dieron a conocer y estudiaron el contenido de las primeras ordenanzas de la tierra de Zamora que se conservan en dicho archivo y que datan de mediados del sigio $\mathrm{x} v$, además ofrecian una inicial aproximación a las relaciones entre los sectores dominantes de la sociedad urbana y la población campesina. Los segundos, agrupados bajo la denominación de Equipo Júpiter, presentaron de forma ordenada y con una breve descripción de cada una, el conjunto de las ordenanzas de Zamora custodiadas en el archivo [27]. Años más tarde, en 1991, y ya con sus autores nominalmente identificados, se publicó la transcripción de todas ellas [19].

De esos momentos data también la catalogación que José Luis Rodríguez de Diego, entonces director del archivo y hoy del General de Simancas, realizó de la documentación medieval conservada en diferentes secciones del mismo (Pergaminos, Desamortización, Hacienda, Municipal y Diputación) y de cuya utilidad hemos sido muchos los beneficiarios $[114,115]$. Ojalá que en un futuro, que esperemos no sea lejano, podamos contar con un catálogo exhaustivo del conjunto de la documentación medieval de este magnífico depósito documental.

También de esta década datan las primeras investigaciones sobre la comunidad judía de Zamora, con aportaciones de Guadalupe Ramos [111], Marciano Sánchez [126] y yo mismo [58]. Más tarde, en el año 1992, se publicaron dos trabajos más sobre la materia elaborados por Florián Ferrero [35] y $M^{a}$ Fuencisla García Casar [45]. Al respecto de esta última obra no puedo dejar de manifestar cierta extrañeza ante el aparente desconocimiento que la autora parece tener de la existencia de algunos de esos trabajos anteriores, algo sorprendente si tenemos en cuenta que los mismos aparecieron en revistas de amplia difusión entre los hebraístas como El Olivo o Sefarad, que la autora reconoce además haber consultado. Esto provoca algunas situaciones que cabria calificar como paradójicas, como son el hecho de que se presente determinada documentación como inédita cuando no lo era, o que se elucubre sobre cuestiones que estaban perfectamente resueltas como, por ejemplo, el destino final de la sinagoga y la construcción de la iglesia de San Sebastián, de la que conocemos hasta el precio de los clavos de las puertas $^{5}$.

Pueden verse las cuentas de la obra en el trabajo señalado con el número 59 en el anexo. Una versión más reducida del mismo se presentó como ponencia en el Primer Congreso de Historia de Zamora: Actas, Tomo 3, pp. 565-577. 
En fin, son cosas que pasan aunque no deberian pasar. En cualquier caso, y mal que nos pese a todos, especialmente al autor del rimbombante prólogo de ese libro, es mucho lo que nos queda por saber de la comunidad judía de Zamora, de sus actividades, de su peso específico en la vida económica de la ciudad, de sus relaciones con la población cristiana y las instituciones municipales, de cómo se produjo la integración social de los numerosos conversos tras la expulsión, etc. Asuntos en los que, a pesar de las periurias documentales, habrá que seguir profundizando en el futuro.

Completa la nómina de publicaciones de la época, la personal síntesis de la historia de Zamora $[13,14]$ realizada por María Luisa Bueno, de la que hasta la fecha han aparecido dos entregas que abarcan los siglos $\mathrm{x}$ al $\mathrm{xIII}$; algunos trabajos mios referidos a fenómenos de marginación social como la prostitución y la esclavitud y a la presencia y participación de la ciudad en instituciones como las Cortes y la Hermandad [54, 56]; y una magnifica incursión en el terreno de la biografía, realizada por Asterio Miguel del Brío, relativa a la figura de Juan de Grado, canónigo de la catedral zamorana a finales del siglo xv [22].

Pero indudablemente, el hito fundamental en el desarrollo de la historiografía zamorana de los últimos tiempos lo constituye la celebración, en marzo de 1988, del Primer Congreso de Historia de Zamora $[3,4]$. No es posible, por razones obvias de espacio y tiempo, reseñar aquí el contenido de la cincuentena de ponencias y comunicaciones presentadas en las distintas secciones del mismo y que, de un modo u otro, tienen como protagonista a la época medieval.

Sin embargo, si creo conveniente señalar las que, a mi juicio, fueron las dos principales aportaciones de aquella reunión. En primer lugar, la presentación, de forma conjunta y sistematizada, de los diferentes archivos y depósitos documentales de utilidad para la investigación sobre el medievo zamorano; mucho más si tenemos en consideración que bastantes de esas primeras aproximaciones se convirtieron, en los años posteriores, en extensas publicaciones, merced al patrocinio del Instituto de Estudios Zamoranos, imprescindibles para el historiador. Entre ellas son de obligada mención la colección diplomática del monasterio de Valparaíso [80], la reseña de la documentación zamorana conservada en el Archivo General de Simancas [122], el catálogo del Archivo del Estado Noble de Zamora [43], el inventario del Archivo del Vizconde de Garcigrande [44] —completado años más tarde con un precioso volumen dedicado a sus pergaminos y libros manuscritos [53]-, o, por último, los catálogos de los archivos municipales de otras localidades de la provincia como Benavente ${ }^{6}$. Tampoco cabe olvidar otra importantísima recopilación documental como es la de los fueros locales de la provincia, realizada por Justiniano Rodriguez [116], de la que también se presentó un primer avance durante el congreso.

${ }^{6}$ Caso Cañibano, M. ${ }^{3}$ Ángeles et alii: El Archivo Municipal de Benavente. Zamora, 1996. Vid. Tb. FerNÁNDEZ RuIz, Raquel: Colección diplomática del monasterio de Santo Domingo de Benavente (12281390). Benavente, 2000. 
En segundo lugar, el hecho de que en el congreso tuvieran cabida algunas líneas de trabajo hasta ese momento nada o escasamente desarrolladas. Por ejemplo, la incidencia de las órdenes militares en tierra zamorana ${ }^{7}$, la evolución institucional del concejo y de las estructuras del gobierno municipal y sus relaciones con otros núcleos e instituciones ${ }^{8}$, la aproximación al estudio de algunos señoríos o de algunos individuos y familias de la oligarquía local, la organización social del espacio en los primeros siglos medievales, cuestiones demográficas ${ }^{9}$, obras públicas y desarrollo urbano, etc. $Y$ también el replanteamiento, desde criterios historiográficos actualizados, de temas clásicos como el archiconocido «motín de la trucha", objeto de dos comunicaciones sumamente interesantes ${ }^{10}$. Al respecto de esta última cuestión, ha aparecido recientemente un trabajo de Fernando Luis Corral en el que, recapitulando lo escrito hasta ahora, sitúa el acontecimiento en su contexto político y analiza el verdadero trasfondo social del enfrentamiento, despojándole de los aditamentos legendarios [87].

Por fortuna, muy al contrario de lo que con demasiada frecuencia sucede, la celebración del Congreso y posterior publicación de sus actas, no implicó una paralización o ralentización de la producción historiográfica sobre Zamora. Cabe atribuir esta circunstancia a la sucesiva convocatoria en la ciudad de eventos en los que la historia medieval tuvo un protagonismo destacado. Me refiero a las Jornadas sobre Fuentes y Métodos de Historia Local [5], a las ya mencionadas sobre Zamora su entorno y América [6] —ambas organizadas por el Instituto de Estudios Zamoranos-, a la reunión en Zamora de la asamblea anual de la Sociedad de Estudios Medievales en 1993 y, por último, a las numerosas actividades generadas por la conmemoración del 1100 aniversario de la refundación de la ciudad por Alfonso III. Entre estas últimas, indudablemente destaca la iniciativa alentada y desarrollada por el profesor Martín de recuperación de los escritos del que podemos considerar primer historiados zamorano: Fray Juan Gil de Zamora en especial, para lo que aqui concierne, las Alabanzas e Historia de Zamora, con traducción y estudio de Jenaro Costas [46]. Al amparo de esa iniciativa se sitúa también la recuperación de los textos cronísticos altomedievales relacionados con Zamora llevada adelante por Fernando Luis Corral [85]. En esa misma línea de rescate de obras clásicas, es también obligada la mención a la edición de la obra de Valeriano Or-

Enrique Rodriguez-Picavea en su comunicación titulada «Las órdenes militares en Zamora durante el siglo xil» se referia a la total ausencia de estudios sobre la presencia de estas instituciones en la provincia de Zamora. En los años posteriores, esta llamada de atención se ha visto respondida con la aparición de algunos trabajos que han comenzado a paliar esta situación. Entre ellos cabe mencionar los de Lera Maillo [81] . Cabezas Lefler [18] y Ferreras Fincias [33] sobre la Orden de Santiago, Ojeda Nieto [102] sobre la de Sain Juan y Barquero Goñi [10] sobre los Hospitalarios.

Jesús I. Coria Colino: "El pleito entre cabildo y concejo zamorano de 1278. Análisis de la conflictividad jurisdiccional. Concejo, cabildo y Rey".

"Manuel PAScual SAnChez: "Aportaciones al estudio de la Historia de la población medieval de Za. mora"

Emesto FERNANDEZ-XESTA: "El motín de la trucha y sus consecuencias sobre don Ponce Giraldo de Cabrera. 'Principe de Zamora'». Esther Pascua Echegaray: "El papel de la nobleza en las relaciones entre Castilla y León a mediados del siglo xil: el caso de Zamora". 
dóñez de Villaquirán sobre la traslación de los restos de San Ildefonso, de la que fue autor Vicente Bécares en 1991 [103].

La aparición en 1995 de la Historia de Zamora editada por el Instituto de Estudios Zamoranos [1], supuso un loable esfuerzo de sintesis y actualización del conocimiento histórico a partir de criterios historiográficos actuales $y$, desde ese punto vista, no cabe sino elogiar la iniciativa, especialmente por lo que implica de acercamiento de la tarea de los historiadores a un público más amplio. Las diez aportaciones que la obra incluye relativas al periodo medieval cumplen con creces esa misión, aunque presenten los lógicos desequilibrios, repeticiones y lagunas en el tratamiento de algunos temas dificilmente evitables en toda obra de carácter colectivo. En mi opinión resulta especialmente elogiable la aportación de Severiano Hernández Vicente, que nos ofrece una utilísima panorámica de las fuentes y archivos de interés para la historia de Zamora, facilitando numerosas referencias concretas de documentación inédita que, sin duda, contribuirán de forma notable al desarrollo de la investigación.

La producción medievalista de estos primeros años noventa, además de los trabajos ya mencionados, se completa con la aparición de un par de excelentes artículos de los que es autor Florián Ferrero; el primero de ellos dedicado a la descripción y análisis del contenido del Libro Tumbo de las Escrituras de Zamora [37], conservado en el Archivo Histórico Provincial, y otro en el que estudia una serie de repartimientos fiscales de la tierra de la ciudad en las postrimerías del siglo xv que le permiten delimitar con precisión la jurisdicción de Zamora y analizar la conflictividad a que da lugar el establecimiento de la misma [36].

Por otro lado, tamibién se publicó nuestra propia tesis doctoral sobre la ciudad en la época de los Reyes Católicos [61], centrada especialmente en la explicación de la articulación y desarrollo de las actividades económicas y el estudio de la evolución de las estructuras del gobierno urbano desde la concesión del regimiento por Alfonso XI. Más recientemente publicamos la transcripción de las Actas de las reuniones del Consistorio de regidores entre 1500 y 1504, las primeras conservadas, que constituyeron uno de los apoyos fundamentales para el desarrollo de dicha investigación [70].

Una investigación que, quizás de forma menos activa, ha seguido dando sus frutos en los últimos años, en los que han aparecido algunos trabajos que deben mencionarse. Asterio Miguel del Brío ha realizado una nueva incursión en el campo de la biografía, en este caso sobre Florián Docampo [24], con una parte preliminar dedicada a la saga familiar de los Docampo que sin embargo, y a mi parecer, no agota las posibilidades de estudio de este linaje de la oligarquía local. Fernando Luis se ha adentrado en un tema muy poco trabajado, como bien se encarga de recordar el autor quizás con una excesiva acritud, el de la infraestructura molinera de la ciudad y su importancia, tanto desde el punto de vista económico como en lo que atañe a las relaciones sociales [86]. Florián Ferrero ha analizado un curioso documento relativo a la profanación de la tumba de Arias Gonzalo en 
tiempos de Enrique IV [38]. Nosotros mismos hemos abordado algunas otras cuestiones relativas a la producción y consumo de vino o las repercusiones del paso por la ciudad de la princesa de Gales en 1501 y el desarrollo de las honras fúnebres de la reina Isabel $[67,69]$.

La obra de Manuel Gómez Ríos sobre Alba de Aliste [50] constituye una primera aproximación al conocimiento de la formación y desarrollo del condado de Alba de Aliste y el papel de los Enriquez en la vida social, política y económica de la ciudad que, como acertadamente ha señalado Severiano Hernández, es uno de los grandes temas pendientes de la historiografía medieval zamorana para cuya elaboración será preciso profundizar en el conocimiento de archivos hasta ahora muy poco utilizados como el ducal de Medina Sidonia o el del condado de Bornos [110], además de la documentación que todavía deben proporcionar los grandes archivos nacionales.

También son de destacar las recientes aportaciones de Iñaki Martín Viso [93, 95] sobre la articulación del espacio y del poder en tierras zamoranas en los siglos altomedievales, materia en la que ya habían incidido anteriormente otros autores como Pascual Martínez [96] o Luis Miguel Villar [132]. Por último, Los trabajos de Fuentes Ganzo [41] sobre la moneda medieval, Gutiérrez González sobre las fortificaciones de la ciudad [52] y Sergio San Marcelo [119] sobre la heráldica y edificios de la ciudad, constituyen tres aportaciones con un alto valor instrumental de imprescindible conocimiento.

Hasta aqui este somero repaso a la historiografía medieval de la ciudad en las últimas décadas. Aunque he procurado ser exhaustivo en la elaboración del anexo bibliográfico que se acompaña, estoy seguro de que por desconocimiento me habré dejado en el tintero la mención de algunas publicaciones relevantes. Pido disculpas a sus autores.

\section{ANEXO BIBLIOGRÁFICO}

\section{LA INVESTIGACIÓN SOBRE ZAMORA EN LA EDAD MEDIA}

1. AA.VV.: Historia de Zamora. Tomo I. De los origenes al final del Medievo. Zamora, IEZ, 1995.

2. AA. VV.: Civitas. MC Aniversario de la ciudad de Zamora. Zamora, 1993.

3. Actas del Primer Congreso de Historia de Zamora. Tomo I. Fuentes Documentales. Zamora, IEZ, 1989.

4. Actas del Primer Congreso de Historia de Zamora. Tomo III. Medieval y Moderna. Zamora, IEZ, 1991.

5. Actas. Fuentes y métodos de la historia local. Zamora, IEZ, 1991. 
6. Actas. Zamora, su entorno y América. Zamora, IEZ, 1992.

7. AlFONSO ANTÓN, Isabel: "Sobre la organización del terrazgo en Tierra de Campos durante la Edad Media", Agricultura y Sociedad 23 (1982), pp. 217-232.

8. Alfonso Antón, Isabel: La colonización cisterciense en la Meseta del Duero. El dominio de Moreruela (Siglos XII-XIV). Zamora, IEZ, 1986.

9. Alvarez Martinez, Ursicinio: Historia general civil y eclesiástica de la provincia de Zamora. Zamora, 1889. (reed. Madrid, Revista de Derecho Privado, 1965).

10. BARquero GoNil, Carlos: Los caballeros hospitalarios durante la Edad Media en España. Burgos, 2003.

11. Bueno Dominguez, M. ${ }^{a}$ Luisa: El monasterio de Santa Maria de Moreruela (1143-1300). Zamora, 1975.

12. Bueno Dominguez, $M .{ }^{a}$ Luisa: "Notas acerca de la estructura agraria en el reino de León en el siglo XII», Hispania 135 (1977), pp. 207-219.

13. Bueno Dominguez, M. ${ }^{a}$ Luisa: Historia de Zamora. Zamora en el siglo $X$. Zamora, 1983.

14. Bueno Dominguez, M. ${ }^{a}$ Luisa: Historia de Zamora. Zamora de los siglos XI-XIII. Zamora, 1988.

15. Bueno Dominguez, M. ${ }^{a}$ Luisa: "Santa María de las Dueñas de Zamora. ¿Beguinas o monjas?: el proceso de 1279". Historia, Instituciones, Documentos 20 (1993), pp. 85-105.

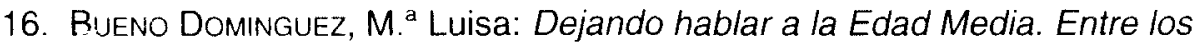
real y lo imaginado. Zamora. Zamora, 1997.

17. Bueno Dominguez, M. ${ }^{a}$ Luisa: Espacios de vida y muerte en la Edad Media. Zamora, 2001.

18. Cabezas Lefler, Carlos: Fuentes documentales para el estudio de la encomienda de Castrotorafe de la Orden Militar de Santiago. Zamora, 1997.

19. Canto de la Fuente, C. el Alii: Ordenanzas municipales de Zamora. Siglos XV y XVI. Zamora, 1991.

20. Coria Colino, Jesús: "Clérigos prestamistas. El mundo de los negocios en una ciudad medieval: Zamora (siglos XIII al XIV)", en El pasado Histórico de Castilla y León I. Edad Media. Actas del I Congreso de Historia de Castilla y León. Burgos, 1983, pp. 343-358.

21. Cruz y MARtin, Ángel: Galeria de zamoranos ilustres. Zamora, 1983. 
22. Del Brio Mateos, Asterio Miguel y Del Brio Carretero, Clara: El canónigo doctor Juan de Grado. Biografía de un clérigo medieval. Madrid, 1987.

23. Del Brio Mateos, Asterio Miguel: Una villa de señorío eclesiástico. Fresno de Sayago (s. XIII-XIX). Zamora, 1986.

24. Del Brio Mateos, Asterio Miguel: La saga de los Docampo y las tierras de Zamora: Alba y Aliste, El Pan, El Vino y Sayago. El maestro Florián Docampo. Zamora, 1997.

25. Del Brio Mateos, Asterio Miguel: Apuntes históricos de Zamora y su tierra. Zamora, 1998.

26. Dominguez Herrero, C.: "Acontecimiento: Cortes en Zamora, 1274, 1301», Anuario del Instituto de Estudios Zamoranos (1988), pp. 347-361.

27. EquiPo JUPITER 81-82: “Ordenanzas de la ciudad de Zamora, siglos XVXVl», Studia Zamorensia 3 (1982), pp. 9-24.

28. Fernandez Dominguez, José: La guerra civil a la muerte de Enrique IV. Zamora, Toro, Castronuño (Reed. Facsimil de la de 1929). Zamora, IEZ, 1993.

29. Fernandez Duro, Cesáreo: Memorias históricas de la ciudad de Zamora, su provincia y obispado. Madrid, 1882.

30. Fernandez Duro, Cesáreo: Colección bibliográfico-biográfica de noticias referentes a la provincia de Zamora o materiales para su estudio. Madrid, 1891 (Reed. Pamplona, Analecta, 2001).

31. Fernandez Prieto, Enrique: Nobleza de Zamora. Madrid, 1953.

32. Fernandez Prieto, Enrique: Apuntes históricos del convento de Santa María la Real de las Dueñas de Zamora. Zamora, 1954 (Reed. 1988).

33. Ferreras Fincias, Francisco J.: "La ermita de Santa Susana de la Orden Militar de Santiago en la ciudad de Zamora", en Las Órdenes Militares en la Península Ibérica. Vol l. Edad Media. Cuenca, 2000, pp. 215-230.

34. Ferrero Ferrero, Florián: VII siglos de Romería a La Hiniesta. Zamora, 1991.

35. Ferrero Ferrero, Florián: Los judios de la provincia de Zamora. Zamora, 1992.

36. Ferrero Ferrero, Florián: "Jurisdicción municipal y poder eclesiástico en la Zamora medieval»", Espacio, Tiempo y Forma- Historia Medieval 7 (1994), pp. 121-158. 
37. Ferrero Ferrero, Florián: «El libro tumbo de las escrituras de la ciudad de Zamora, de los siglos XIV al XVI», Studia Zamorensia I (1994), pp. 97-141.

38. Ferrero Ferrero, Florián: «De la profanación de tumbas en tiempos de Enrique IV y de otros asuntos", en Homenaje a Antonio Matilla Tascón. Zamora, 2002, pp. 185-205.

39. Ferrero Ferrero, Florián et Alii: "Documentación medieval en los Archivos Históricos Provinciales. En el caso del AHP de Zamora», en Fuentes y Métodos de la Historia Local. Zamora, IEZ, 1991, pp. 59-67.

40. FLYNN, Maureen: Confraternal Piety in Zamora in the Late Medieval and Early Modern Period. Ann Arbor, Michigan, 1985.

41. Fuentes Ganzo, Eduardo: "Breve historia de la moneda en Zamora (6121474)», Studia Zamorensia IV (1997), pp. 45-64.

42. Gacto Fernandez, M. ${ }^{a}$ T.: Estructura de la población de la Extremadura Leonesa en los siglos XII y XIII (Estudio de los grupos socio-jurídicos a través de los fueros de Salamanca, Ledesma, Alba de Tormes y Zamora). Salamanca, 1977.

43. Garcia Alvarez, Pedro: Catálogo del Archivo del Estado Noble de la ciudad de Zamora. Zamora, IEZ, 1990.

44. García Alvárez, Pedro y López Alonso, Rosa M. : Inventario del Archivo del vizconde de Garcigrande. Zamora, IEZ, 1991.

45. Garcia Casar, M. ${ }^{a}$ Fuencisla: El pasado judio de Zamora. Valladolid, 1992.

46. GIL DE ZAMORA, Juan: Alabanza e historia de Zamora. (Traducción y estudio de Jenaro Costas Rodríguez). Zamora, 1994.

47. Granja Alonso, Manuel de la: Estudio histórico, artístico, religioso, agricola y humano del Real Monasterio de Santa Maria de Moreruela de la Orden Cisterciense. Zamora, 1990.

48. Granja Alonso, Manuel de la: «Repoblación de Zamora en la Edad Media», Anuario del Instituto de Estudios Zamoranos (1995), pp. 435-466.

49. Gomez Moreno, Manuel: Catálogo monumental de España. Provincia de Zamora. 2 vols. León, 1980 (reed. Facsimil de la de 1927).

50. Gomez Rios, Manuel: Alba de Aliste (1190-1564), el castillo, el señorío, el condado. Roma, 1997.

51. Guadalupe Beraza, M. ${ }^{2}$ Luisa: “El tesoro del cabildo zamorano: aproximación a una biblioteca del siglo XIII», Studia Histórica Medieval I-2 (1983), pp. 167-180. 
52. GutiérRez González, J. A.: “Las fortificaciones de la ciudad de Zamora. Estudio arqueológico e histórico". Cuadernos de Investigación Florián de Ocampo 6. Zamora, 1990.

53. Hernandez Hernandez, Gerardo: Pergaminos y libros manuscritos del Vizconde de Garcigrande (1421-1881). Archivo de la Diputación de Zamora. Zamora, 1998.

54. Ladero QueSADA, Manuel Fernando: “Sobre la marginación social en Zamora a finales de la Edad Media: prostitución, pobreza y esclavitud". Anuario del Instituto de Estudios Zamoranos (1986), pp. 213-220.

55. Ladero Quesada, Manuel Fernando: "Notas sobre las propiedades del Cabildo catedralicio de Zamora en el último tercio del siglo XIV (13721402)". En la España Medieval 5-l (1986), pp. 537-549.

56. LADERO QueSADA, Manuel Fernando: "La participación de Zamora en instituciones de ámbito general de la Corona de Castilla: Las Cortes y La Hermandad (siglo Xv)". Anuario de Estudios Medievales 18. Estudios dedicados a la memoria del Profesor Emilio Sáez (1988), pp. 399-408.

57. Ladero QueSada, Manuel Fernando: «Aproximación al proceso de formación del patrimonio de la Iglesia zamorana (obispo y cabildo), 11321484". Espacio, Tiempo y Forma, Serie III, nº 1 (1988), pp. 249-269.

58. Ladero Quesada, Manuel Fernando: “Apuntes para la historia de los judíos y los conversos de Zamora en la Edad Media (siglos XIII-XV)", Sefarad XLVIII, 1 (1988), pp. 29-57.

59. LADERO QUESADA, Manuel Fernando: "La remodelación del espacio urbano de Zamora en las postrimerías de la Edad Media (1480-1520)". Espacio, Tiempo y Forma, Serie III, $\mathrm{n}^{\circ} 2$ (1989), pp. 161-188.

60. Ladero QueSADA, Manuel Fernando: “El concejo de Zamora en el siglo $\mathrm{XV}$ : monopolio y oligarquización del poder municipal. Aproximación al proceso". Espacio, Tiempo y Forma, Serie III, n 3 (1990), pp. 83-93.

61. LaDERo QueSADA, Manuel Fernando: La ciudad de Zamora en la época de Ios Reyes Católicos. Economía y gobierno. Zamora, 1991.

62. Ladero Quesada, Manuel Fernando: "Metodología sobre los estudios de las ciudades medievales en la Corona de Castilla. El modelo de Zamora". Actas de las I Jornadas sobre Fuentes y Métodos de la Historia Local (Zamora, 1990). Zamora, Instituto de Estudios Zamoranos 1991, pp. 47-55.

63. LADERO QUeSADA, Manuel Fernando: “Zamora en las postrimerías de la Edad Media: una visión panorámica". Actas de las I Jornadas sobre Zamora, su entorno y América (Zamora, 1991). Zamora, Instituto de Estudios Zamoranos 1991, pp. 33-42. 
64. Ladero Quesada, Manuel Fernando: “Judios y cristianos en la Zamora medieval'. Actas del Congreso 'La proyección Histórica de España en sus tres culturas (Medina del Campo, 1991), vol. I. Valladolid, 1993, pp. 159-164.

65. Ladero Quesada, Manuel Fernando: «llustraciones sobre Zamora a comienzos de la modernidad según las actas de las reuniones del Consistorio (1500-1504)». Studia Zamorensial (1994), pp. 143-158.

66. Ladero Quesada, Manuel Fernando: «Bandos ciudadanos en la Zamora medieval: oligarquia y común", en 893-1993, Zamora 1100 años de Historia (Ed. José Luis Martín). Zamora, 1995, pp. 61-78.

67. LADERO QUESADA, Manuel Fernando: "Sobre el viñedo y el vino en Zamora y su tierra a finales de la Edad Media». Studia Zamorensia II (1995), pp. 27-47.

68. LADERO QUESADA, Manuel Fernando: "Zamora: formulación y dinámica del poder en un concejo medieval’. Medievalismo. Boletín de la Sociedad Española de Estudios Medievales n ${ }^{\circ} 6$ (1996), pp. 147-160.

69. Ladero QueSADA, Manuel Fernando: «Recibir princesas y enterrar reinas (Zamora 1501 y 1504)». Espacio, Tiempo y Forma, Serie III, $n^{\circ} 13$ (2000), pp. 119-137.

70. Ladero Quesada, Manuel Fernando: Libros de acuerdos del consistorio de la ciudad de Zamora (1500-1504). Zamora, 2000.

71. LARRÉN IZQUIERDO, Hortensia: "La evolución urbana de la ciudad de Zamora a través de los vestigios arqueológicos", en El urbanismo de los estados peninsulares. Aguilar de Campoo, 1999, pp. 91-118.

72. LeRA Malllo, José Carlos: "La documentación medieval del archivo catedral de Zamora: fuentes para la historia de Zamora", en Fuentes y Métodos de la Historia Local. Zamora, IEZ, 1991, pp. 69-80.

73. Lera Malllo, José Carlos: "El cartulario del siglo XIII del monasterio de Valparaiso", Memoria Ecclesiae VII (1995), pp. 293-302.

74. LeRA Malllo, José Carlos: "Los libros de visitas pastorales medievales en el Archivo Histórico Diocesano de Zamora. Presentación y estudio diplomático". Memoria Ecclesiae VIII (1996), pp. 499-508.

75. LeRa Malllo, José Carlos: “Las cartas de obediencia de los abades de Valparaíso al obispo Suero de Zamora. Estudio diplomático", IX Centenario de la Fundación del Císter. Actas del II Congreso Internacional SCbre el Cister en Galicia y Portugal. Vol. I. Orense, 1998, pp. 497-503.

76. Lera Maillo, José Carlos: Catálogo de los documentos medievales de la catedral de Zamora. Zamora, IEZ, 1999. 
77. Lera Maillo, José Carlos: «El proceso de fundación y consolidación del monasterio de Santa María de Valparaíso", Anuario del Instituto de Estudios Zamoranos (2001). pp. 337-347.

78. LeRA MAILLO, José Carlos: "La Iglesia de Zamora y sus relaciones con Roma en el siglo XII’, Studia Zamorensia VI (2002), pp. 9-28.

79. Lera Malllo, José Carlos: «El testamento del obispo Pedro II. Año 1302. Edición diplomática», Homenaje a Antonio Matilla Tascón. Zamora, 2002, pp. 355-360.

80. Lera Mallzo, José Carlos et alii: Colección diplomática del Imperial Monasterio de Nuestra Señora de Valparaíso (1143-1499). Zamora, IEZ, 1998.

81. Lera Maillo, José Carlos y Turiño Mínguez, Ángel: La orden militar de Santiago en la provincia de Zamora. Edición diplomática de la visita a las encomiendas de Castrotorafe y Peñausende. Año 1528. Zamora, 2000.

82. LINEHAN, Peter: La Iglesia española y el papado en el siglo XIII. Salamanca, 1975.

83. Lineman, Peter: The Ladies of Zamora. Manchester University Press, 1997. (traducción al español: Las dueñas de Zamora: secretos, estupro y poderes en la iglesia española del siglo XIII. Barcelona, Ed. Península, 1999).

84. LINEHAN, Peter y LeRa MaIllo, José Carlos: Las postrimerías de un obispo alfonsino. Don Suero Pérez, el de Zamora. Zamora, 2003.

85. LUIS CoRRAL, Fernando: Zamora, de las crónicas al romancero. Salamanca, 1993.

86. LuIS CorRaL, Fernando: «Feudalismo y molinos: la posesión de aceñas en Zamora en el siglo XII", Studia Zamorensia III (1996), pp. 53-75.

87. LUIS CORRAL, Fernando: «Leyenda y realidad histórica: el contexto político del Motín de la Trucha de Zamora en el siglo XII", Studia Zamorensia VI (2002), pp. 29-47.

88. Malllo Salgado, Felipe: Zamora y los zamoranos en las fuentes arábigas medievales. Salamanca, 1990.

89. Martín Rodriguez, José Luis: Documentos Zamoranos. 1. Documentos del Archivo Catedralicio de Zamora. Primera parte (1128-1261). Salamanca, 1982.

90. Martin Rodriguez, José Luis: Amor, cuestión de señorío y otros estudios zamoranos. Zamora, UNED, 1993. 
91. Martin Rodriguez, José Luis (ed): 893-1993. Zamora 1100 años de historia. Zamora, 1995. "Fueros y contratos agrarios: el fuero de Zamora", pp. 35-44

92. MARTIN VISO, Iñaki: «Una comarca periférica en la Edad Media: Sayago, de la autonomía a la dependencia feudal», Studia Histórica-Historia Medieval 14 (1996), pp. 97-155.

93. MARTIN VISO, Iñaki: "La articulación del poder en la cuenca del Duero: el ejemplo del espacio zamorano (siglos VI-x)", Anuario de Estudios Medievales 31/1 (2001), pp. 75-126.

94. MARTIN VISo, Iñaki: "La formación de la frontera con Portugal y su impacto en el occidente zamorano (s. XII-XIII)", Studia Zamorensia VI (2002), pp. 49-74.

95. Martin Viso, Iñaki: Fragmentos de Leviatán: la articulación del espacio zamorano en la Alta Edad Media. Zamora, IEZ, 2002.

96. Martinez Sopena, Pascual: La Tierra de Campos occidental. Poblamiento, poder y comunidad del siglo $x$ al XIII. Valladolid, 1985.

97. Mateos Carretero, José Antonio: Andavias, un lugar de realengo en la jurisdicción de Zamora. Siglos XII al XIX. Madrid, 1994.

98. Matilla Tascón, Antonio: Guía-inventario de los archivos de Zamora y su provincia. Madrid, 1964.

99. Moreno Sebastián, Atilana: Los señorios de la iglesia en la tierra de Zamora, siglos XVI-XIX. Los procesos desamortizadores de la riqueza señorial. Zamora, 1984.

100. Moreno Sebastian, Atilana: Conflictos jurídicos en la abolición de los señorios de la grandeza de Zamora. Prestaciones subsistentes hasta la Reforma Agraria de la II República. Zamora, 1993.

101. Moreta, S. y VACA, A.: "Los concejos urbanos: núcleos de señorios corporativos conflictivos", Agricultura y Sociedad 23 (1982), pp. 343-385.

102. OJeda Nieto, José: Comendadores y vasallos. La Orden de San Juan y el partido de Valdeguareña. Zamora, 1997.

103. Ordoñez de VILLAQuirán, Valeriano: La translación de San Ildefonso (1496). (Edición, transcripción y traducción de Vicente Bécares Botas). Zamora, 1991.

104. Pérez Bustamante, Rogelio y Ferrero Ferrero, Florián (coord): Historia de Zamora. Valencia, 1990.

105. Pérez Martin, A.: “Estudiantes zamoranos en Bolonia», Studia Zamorensia 2 (1981), pp. 23-66. 
106. Pescador del Horo, $M^{a}$ Carmen: Archivo Municipal de Zamora. Documentos históricos. Zamora, 1948.

107. Pescador del Hoyo, $M^{a}$ Carmen: El santo rey Fernando III y su tierra de Zamora. Zamora. 1953.

108. Pescador del Hoyo, $M^{a}$ Carmen: "Los gremios artesanos de Zamora", Revista de Archivos, Bibliotecas y Museos LXXV (1968-1972), pp. 183200; LXXVI (1973), pp. 13-60; LXXVII (1974), pp. 67-101 y 449-520; LLLLXXVIII (1975), pp. 605-691.

109. Pescador del. Hoyo, $M^{a}$ Carmen: "El tesoro del Monasterio de Montamarta», En la España Medieval 5-il (1986), pp. 831-847.

110. Porras Arboledas, Pedro: «Inventario del Archivo del Conde de Bornos", Espacio, Tiempo y Forma, Serie III n 8 (1995), pp. 183-289.

111. Ramos de Castro, Guadalupe: Juderias de Castilla y León. Zamora, 1988.

112. Represa, Amando: "Génesis y evolución urbana de la Zamora medieval», Hispania 122 (1972), pp. 525-545.

113. Revuelta Somalo, José María: Los Jerónimos. Una orden religiosa nacida en Guadalajara. Guadalajara, 1982.

114. Rodriguez DE DiEgo, José Luis: "Documentación medieval del Archivo Histórico Provincial de Zamora», Studia Histórica-Ha Medieval l-2 (1983), pp. 181-208.

115. Rodriguez DE DIEGo, José Luis: «Documentos medievales conservados en el Archivo Histórico Provincial de Zamora", Studia Zamorensia 4 (1983), pp. 9-34.

116. Rodriguez Fernandez, Justiniano: Los fueros locales de la provincia de Zamora. Salamanca, 1990.

117. Rodriguez Gonzalez, A.: El Tumbo del monasterio de San Martín de Castañeda. León, 1973.

118. Rodriguez Rodriguez, Elías: "El monasterio de Moreruela y el concejo de Villafáfila: historia de un conflicto secular", Anuario de Instituto de Estudios Zamoranos (2002), pp. 277-321.

119. San marcelo y Vassallo, Sergio Jesús de: Heráldica y edificios de Zamora. Zamora, 1998.

120. Sanchez Herrero, José: "Cofradías, hospitales y beneficencia en algunas diócesis del valle del Duero, siglos XIV y XV», Hispania 126 (1974), pp. $5-51$. 
121. Sanchez Herrero, José: "La Iglesia y la religiosidad en la Diócesis de Zamora a finales del siglo XV», en Zamora, su entorno y América. Zamora, IEZ, 1992, pp. 43-94.

122. Sanchez Rivera, $M^{a}$ Luisa: Fuentes documentales zamoranas en el Archivo de Simancas. Zamora, IEZ, 1990.

123. Sanchez Rooriguez, Marciano: "Una cláusula penal del Tumbo Negro de Zamora", Homenaje a Fray Justo Pérez de Urbel I, Silos, 1976, pp. 339 379.

124. Sanchez Rodriguez, Marciano: Tumbo blanco de Zamora. Salamanca, 1985.

125. Sanchez Rodriguez, Marciano: "Una reglamentación de aceñas de Zamora (siglo XIV)», Studia Zamorensia 6 (1985), pp. 61-86.

126. Sanchez Rodriguez, Marciano: "Judíos zamoranos: reflexiones en torno a una historiografía», El Olivo 23 (1986), pp. 11-38.

127. Sanchez Rodriguez, Marciano: "Minifundismo y éxodo rural: el caso de Villamor de los Escuderos (siglo Xill)", Studia Zamorensia 7 (1986), pp. 447-464.

128. Sanchez Rodriguez, Marciano: "La venganza de la sangre en Zamora (siglo xIII)", Studia Zamorensia 8 (1987), pp. 93-104.

129. Sanchez Rodriguez, Marciano: Fueros y posturas de Zamora (Tumbos Blanco y Negro). Salamanca, 1987.

130. Sanchez Rodriguez, Marciano: "Motes, remoquetes o apellidos en el Tumbo Negro de Zamora. Datos para una sociolingüística aplicada", Studia Zamorensia IV (1997), pp. 205-234.

131. VACA LORENZO, Ángel: Documentación medieval del monasterio de Santa Clara de Villalobos (Zamora). Salamanca, 1991.

132. VILLAR GarCiA, Luis M.: "Ocupación y organización social del espacio", en 893-1993. Zamora 1100 años de Historia (ed. J.L. Martín), Zamora, 1995, pp. 51-60.

133. Zaragoza Pascual, E.: "Los monasterios benedictinos en la ciudad de Zamora", Nova et Vetera 10 (1980), pp. 267-291.

134. Zaragoza Pascual, E.: San Martín Cid. Fundador y primer abad del monasterio cisterciense de Valparaíso. Zaınora, 1980. 